\title{
XXX.ON THE PROPERTIES OF PURE METALLIC ALUMINUM.
}

\section{By J. W. Mallet.}

In view of the marked effect upun the properties of the individual metals, which is often produced by the presence of even very small quantities of foreign substances, metallic or non-metallic, it is very desimble that when really pure specimens can be had, prepared and tested with unusual care, their properties shall be noted and recorded. Comparatively few of the metals have probably been procured and examined in a state of the highest attainable purity, and comparatively few chemists bave learned by personal experience how very difficult it is to get rid of the last detectable traces of foreign matter. This difficulty is especially great in the case of those metals least easily reduced to the metallic state, among which may be counted aluminum.

The writer, having expended much time and labor upon obtain. ing pure metallic aluminum to be used in his work*, on the atomic weight of this element, succeeded in preparing enough for that purpose, and incidentally noted the following facts in regard to the metal一not forming a complete description of all its properties, physical and chemical-but yet deserving, perhaps, of being placed on record.

The crude (commercial) aluminum, used as the source of the pure metal, was found to contain:

Aluminum (by difference) ................. 96.89

Iron ................................

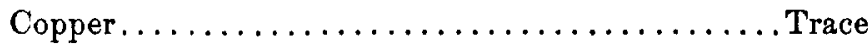

Silicon.............................27

100.00

This was converted into bromide by treatment with liquid bromine, observing the precautions described in the paper (in Phil. Trans.) above referred to. A large quantity of the aluminum bromide-more than two kilogrammes altogether-was purified by careful fractional distillation, with due regulation of temperature, repeater a number of times, until the product was perfectly colorless, and on solution in water gave no evidence of the presence of

- Philnsophical Transactions-Pt. III., 1880. 
impurities, which were searchingly tested for. The reduction of the metal from this purified bromide was accomplished, but with much difficulty and great loss, by heating with pure sodium in a crucible made of, or lined with, a mixture of purified and $d r y$ alumina and sodium aluminate. The large globules of aluminum obtained were severally re-fused before the blowpipe on a support of alumina, and were then exposed for a short time to the action of hydrochloric acid on the surface, well washed with water, and dried. On solution of portions of the globules in pure hydrochloric acid, careful testing failed to show the presence of any of the impurities which might have been taken up from the materials and apparatus need. The entire absence of sodium was specially verified.

The color of this fure aluminum was perceptibly whiter than that of the commercial metal-on a cut surface very nearly pure tin-white, without bluish tinge, so far as could be judged from the small pieces examined. The lustre, too, was very much that of tin in a fresh, untarnished condition.

The metal was distinctly softer than before puritication. Hence its fracture was not easily observed, but seemed to be very fine grained, with sume appearanee of tibrous silkiness. The malleability was undoubtedly improved, the metal yielding easily to the hammer, and bearing distortion well by flattening in two or three directions without cracking. It seemed to be sensibly less hardened by bammering than the ordinary metal of commerce.

The specific gravity was carefully determined at $4^{\circ} \mathrm{C}$., and the mean of three closely concordant experimental results gave the number 2.583 as referred to water at the same temperature.

Hence the "atomic" volume $=10.45$, near that of gold $(10.19$, if the sp. gr. be taken as 19,295 , the value found by Roberts for the large trial-plate of fine gold of the English mint), and standing to that of iron nearly as $3: 2$ (the at, vol. of the latter being 7.11 , if the $\$$. gr. be taken $=7.88$, as found by Caron for pure iron fused in the oxy-hydrogen flame).

The specific heat was determined by means of Bunsen's ice calori. meter and found to be $=.2253$ as a mean for the range of temperature $0^{\circ}-100^{\circ}$ C., a number rather bigher than those obtained by Regnault and Kopp. This number multiplied by 27.02, the atomic weight found in the experiments for which the metal was prepared, gives the product 6.09 as the atomic heat.

The quantity of pure material at command was not sufticient to 
allow of any determination of the conductivity, though this would be an interesting point to examine, in view of the high conducting power of the commercial metal, and the probability that the value would be notably raised by the removal of impurities. An imperfect attempt was made to measure the expansion by change of temperature, using for the purpose determinations of specific gravity in water at widely different temperatures, and correcting for expansion of water and glass, but the quantity of material was too small to make the result trustworthy.

It was also attempted to estimate roughly the fusibility, by placing equally heavy beads of the pure and the commercial aluminum in front of a fine blowpipe jet, this jet being fixed in position and fed with air by a steady water blast. From the difference of distance at which the beads could be just fused it seemed probable that the pure metal was a little less fusible than that of commercial character

Under the same conditions of heating the former seemed to oxidize on the surface with rather more ease than the latter. On the other hand, the pure metal seemed to present perceptibly greater resistance to the prolonged action of solvents-acids and alkaliesthan the impure.

Univ. of Virginia,

Jibly 15, 1882.

CONTRIBUTIONS FROM THE LABORATORIES OF THE SCHOOT, OF MINES, COLUMBIA COLLEGE.

XXXI.-ON NITRO-SACCHAROSE.

By Arthur H. Ellioti, Ph. B., F, C. S.

This curious body has already been investigated by Sobrero in $1847,{ }^{*}$ Schoenbein, $\dagger$ Ad \& W. Knop $\downarrow$ and others; and no definite conclusions as to its constitution appear to have been brought out by their labors. Having to make some experiments upon this body I thought it would not be uninteresting to give an account of some results which $I$ obtained in the course of my work. These experiments include the action of a number of solvents, the action

* Comptes Rendus Vol. 24, p. 247.

† Pogg. Ann. Vol. 70, p. 100.

‡ Jour. fur prak. Chem, Vol. 56, p. 334 . 